Pixel Imaging Mass Spectrometry with fast and intelligent Pixel detectors

This article has been downloaded from IOPscience. Please scroll down to see the full text article.

2010 JINST 5 C07007

(http://iopscience.iop.org/1748-0221/5/07/C07007)

View the table of contents for this issue, or go to the journal homepage for more

Download details:

IP Address: 81.151.194.97

The article was downloaded on 17/08/2010 at 22:25

Please note that terms and conditions apply. 


\section{Pixel Imaging Mass Spectrometry with fast and intelligent Pixel detectors}

A. Nomerotski, ${ }^{a, 1}$ M. Brouard, ${ }^{b}$ E. Campbell, ${ }^{b}$ A. Clark, ${ }^{c}$ J. Crooks, ${ }^{c}$ J. Fopma, ${ }^{a}$

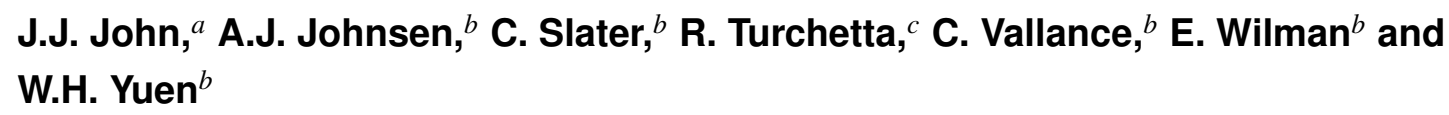

E-mail: A.Nomerotski@physics.ox.ac.uk

ABSTRACT: We report on 'proof of concept' experiments in Pixel Imaging Mass Spectrometry (PImMS) using an ultra-fast frame-transfer CCD camera and also describe an intelligent CMOS sensor which is being developed for this application by the PImMS collaboration in the UK. PImMS is a combination of traditional TOF mass spectrometry and ion imaging. Information provided by the ion imaging gives access to valuable structural information of the molecule under investigation, in addition to the normal mass spectrum. Recording of the 2D spatial information of the arriving ions allows to reconstruct the ion velocity distributions for separate ion masses and to correlate them to each other. The new PImMS sensor will be capable of time stamping up to four arriving ions per pixel during the $200 \mu \mathrm{sec}$ acquisition cycle with $100 \mathrm{nsec}$ resolution which should meet the demanding requirements of complete recording of mass spectra of complex organic molecules.

KEYWORDS: VLSI circuits; Mass spectrometers; Instrumentation and methods for time-of-flight (TOF) spectroscopy; Ion identification systems

\footnotetext{
${ }^{1}$ Corresponding author.
} 


\section{Contents}

1 Introduction 1

2 Pixel Imaging mass Spectroscopy (PImMS) 2

3 Fast framing CCD camera 2

4 CMOS based sensors $\quad 3$

5 PImMS sensor 4

6 Summary 5

\section{Introduction}

Time Of Flight Mass Spectrometry (TOF-MS) is a widely used tool in analytical chemistry. It is based on the principle that ions can be separated according to their mass by recording their arrival times at a detector following passage through a flight tube. In a commercial mass spectrometer, the range of ion flight times is of the order of 100-200 $\mu$ sec. Traditionally, TOF-MS instruments simply measure ion current as a function of arrival time. The technique of velocity/spatial-map ion imaging has the potential to take mass spectrometry beyond this single dimension by associating positional information on the ions within each mass peak. This method is widely used in physical chemistry for analysis of simple molecules [1].

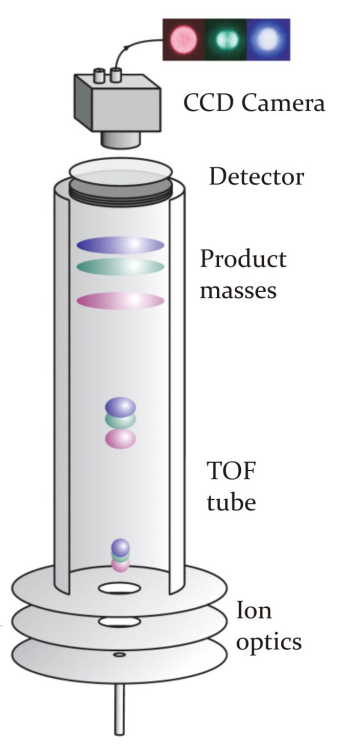

Figure 1. Schematic view of an ion imaging apparatus.

A schematic view of an ion imaging apparatus is shown in figure 1. For photofragment velocity imaging, the parent molecule is prepared in a molecular beam, intercepted by a pair of laser pulses, the first of which fragments the molecule, and the second of which ionizes the resulting fragments. The nascent ions have speed and angular distributions characteristic of the specific fragmentation process in which they were formed. A carefully tuned electrostatic field maintained within the ion optics assembly accelerates the ions along a flight tube towards a position sensitive detector consisting of a pair of microchannel plates (MCPs) coupled to a phosphor screen. All ions of a given mass with a given velocity component in the image plane are mapped onto the same point on the detector, regardless of the position at which they were formed. The image on the phosphor is captured by a camera. To record an image for a single mass, either the MCPs or the camera are gated to the flight time of the ion of interest. Until very recently it has not been possible to image more than one mass per laser cycle due to slow image transfer times. 

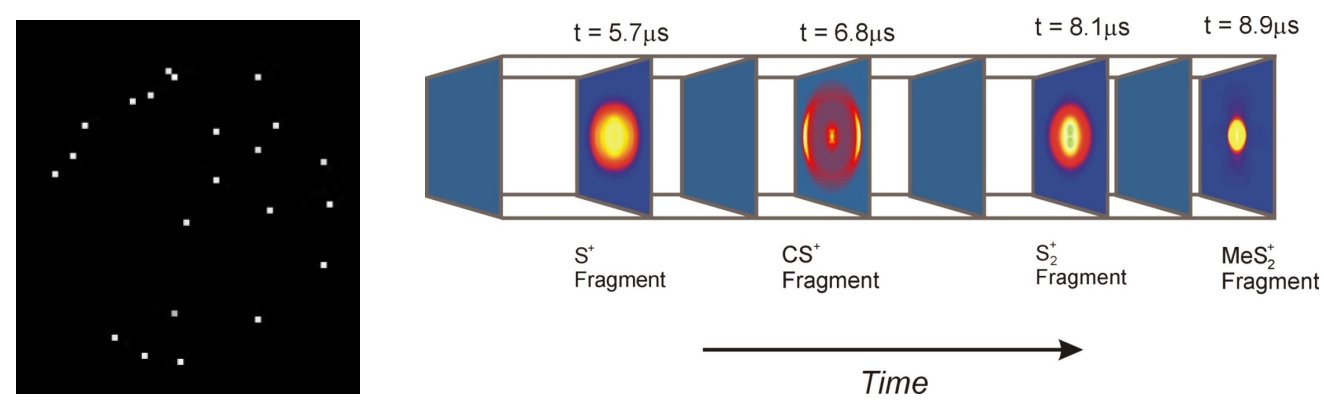

Figure 2. Left: A typical single frame shot taken with the DALSA camera. Right: Velocity maps of fragment ions from the photolysis of dimethyldisulfide resolved for each mass peak.

\section{Pixel Imaging mass Spectroscopy (PImMS)}

With recent progress in silicon technologies, several novel types of fast, position sensitive detectors are becoming available. These overcome the single mass peak limitation and allow imaging of multiple masses in a single acquisition. This approach, Pixel Imaging Mass Spectrometry (PImMS) [2], gives access to new information and provides scope for a range of new and exciting techniques. The PImMS mass resolution is largely determined by the speed of the imaging sensor and the decay lifetime of the phosphor. Below we describe two different technologies appropriate for this application: fast framing CCD cameras and CMOS cameras.

\section{Fast framing CCD camera}

The technology currently in use in our experiments is the Zenith fast framing CCD camera from Dalsa [3], which allows recording of 16 sequential images at $64 \times 64$ resolution with $100 \times 100$ $\mu \mathrm{m}^{2}$ pixels. The maximum frame rate, $100 \mathrm{MHz}$, allows for adjustable image exposure in the range from $10 \mathrm{~ns}$ to $1.2 \mathrm{~ms}$ in $5 \mathrm{~ns}$ increments [4]. The camera is based on the principle of local charge storage in a CCD register at imaging pixel level [5]. After all 16 register cells are full, the recorded information must be read out before image acquisition can continue. The main limitation of the DALSA camera is the small number of frames. The future development of this technology may allow a larger frame number but will always be limited by the pixel area [6].

The first PImMS proof-of-concept experiments were successfully performed in 2008 using the Dalsa Zenith camera. Images were recorded of several photofragments from the $193 \mathrm{~nm}$ photolysis of dimethyldisulphide, a small organic molecule [7]. A typical single frame shot taken with the DALSA camera is shown in the left image of figure 2. Each bright pixel on the image corresponds to detection of a single ion. The ion spatial distributions from these experiments are presented in the right image of figure 2, which shows images, integrated over many frames, for all four detected fragments. The typical separation in arrival times between consecutive fragments in this experiment was about $1 \mu \mathrm{sec}$. The spatial distributions recorded at the detector were used to reconstruct associated speed and angular distributions separately for each TOF mass peak. In addition to providing information on the fragmentation dynamics, the method may also be used to enhance mass fingerprinting, and to deconvolute multiple contributions to a single mass peak. 
Mass 13

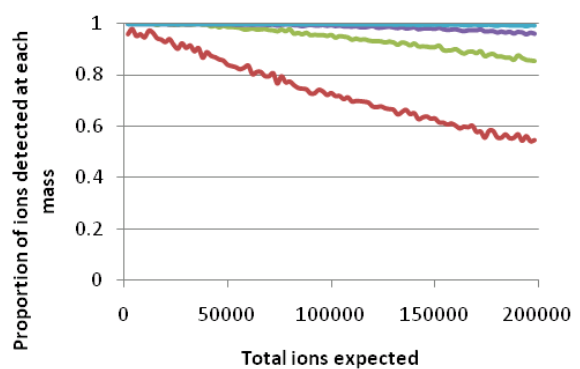

Mass 39

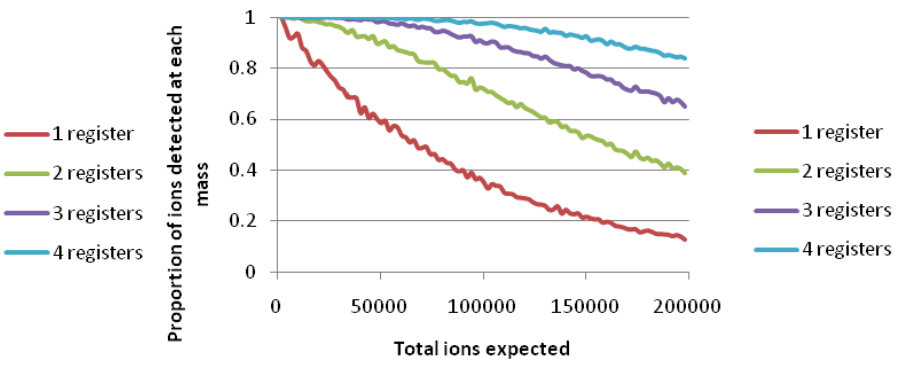

Figure 3. Efficiency to register an incoming ion as a function of the number of ions flown in the apparatus for different number of registers in the pixel.
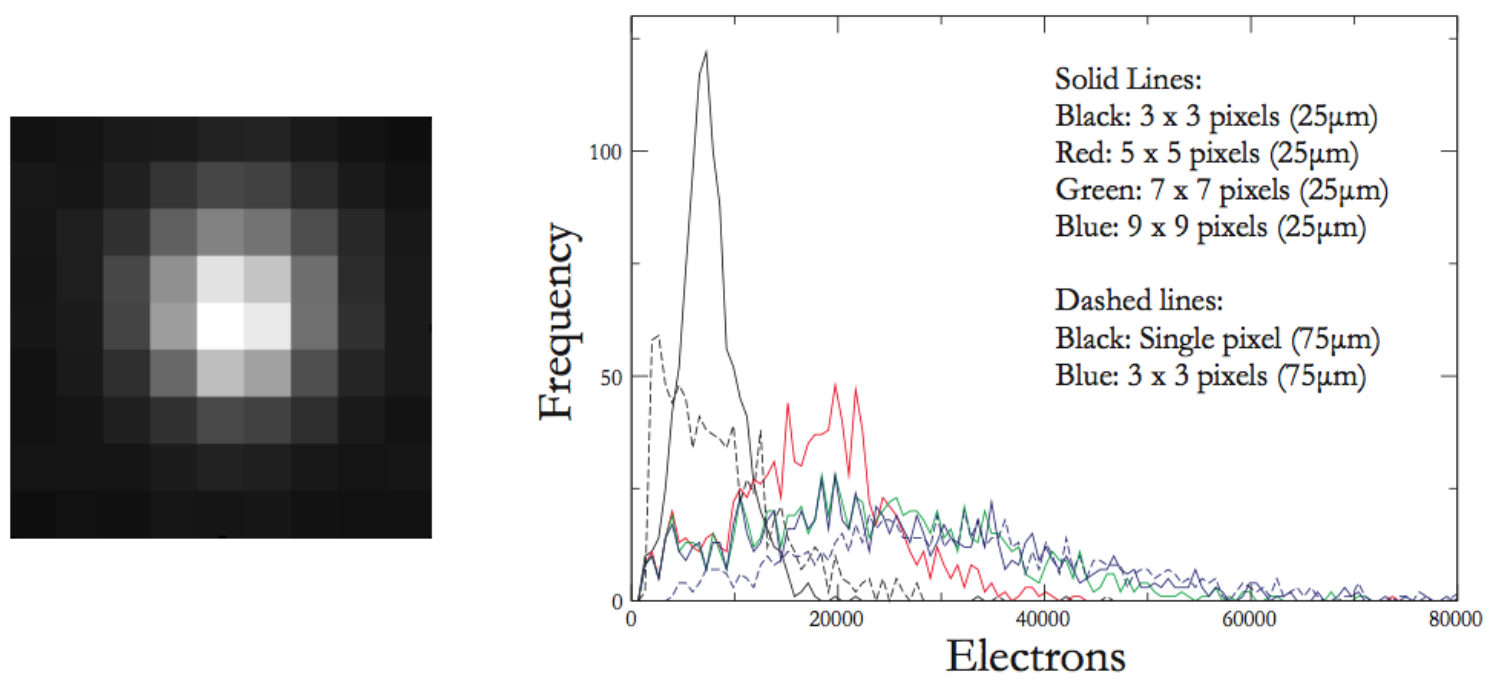

Figure 4. Left: Single cluster in VANILLA. Right: Distribution of cluster charge.

\section{CMOS based sensors}

Technology in CMOS-based image sensing is a rapidly growing area. The CMOS approach allows enhanced in-pixel signal processing and more flexible readout architectures when compared with CCD sensors. For example, a considerable data reduction can be achieved by incorporating 'time stamping' into each pixel, in which time information is recorded only if a pixel registers a light pulse. At low event intensities the time stamp approach provides the same information as full frame readout, while generating much less data. In addition, the intensity requirement can be relaxed if each pixel can record several time stamps.

A simulation study has been performed to investigate the intensity limitation. Forty ion masses of equal intensities were generated, each with a uniform velocity distribution. The ions were propagated through a TOF-MS onto a $256 \times 256$ pixel sensor.

The efficiency to register an incoming ion as a function of the number of ions flown in the apparatus is shown in figure 3 for options with one, two, three and four time stamp registers in the pixel. It was assumed that an ion strike only triggers one pixel, the pixel remains sensitive until all 
its registers are filled up, and that a typical experiment produces up to 200000 ions. For ion strikes triggering several pixels, the maximum number of ions detected is decreased pro rata. The left graph in figure 3 corresponds to one of the early ions while the right graph corresponds to the second last ion. The efficiency is lower in the latter case as the previously arrived ions deplete the number of available registers. The case with four registers offers considerable advantages, especially for heavier ions in the end of the mass spectrum. Where ions are not detected through saturation, the mass spectrum generated is distorted and the image produced has areas of decreased detection efficiency due to previous ions. Four registers appears to eliminate saturation effects in almost all cases, allowing one to run at nominal ion intensities without compromising the data collected.

In a CMOS pixel sensor (monolithic active pixel sensor or MAPS) the light is detected in a thin epitaxial layer near the surface, producing charge which is collected by diffusion to an $n+$ node. Normally, the pixel has limited functionality, as only NMOS type transistors are allowed [10]. The INMAPS process developed at the Rutherford Appleton Laboratory, shields the PMOS type transistors with a deep $p+$ implant, resulting in better charge collection efficiency and enabling the full CMOS capability [11]. The INMAPS process has already been used in a number of projects in particle physics and for other applications [12].

\section{PImMS sensor}

The INMAPS process described above is currently being used to produce a new specialized sensor for PImMS. The sensor will have $512 \times 512$ pixels with dimensions $70 \times 70 \mu \mathrm{m}^{2}$. The main function of the sensor is to record time stamps for ions which produce a light signal more than a predefined threshold. During the measurement period the sensor generates a time-code which is distributed to all pixels. The pixel stores the time-code in one of four 12-bit memories in the pixel at the point in time when an event occurs. After the experiment is complete all memory locations are read out and transferred to a PC. After readout the sensor can be reset and is capable of repeating the experiment at up to 20 times per second. It is worth mentioning that the 12-bit counter will effectively correspond to 4096 time slices. This will dramatically increase the potential number of mass peaks that may be recorded compared to the fast framing CCD camera currently in use, and will allow the sensor to be applied to studies of complex organic molecules.

To define the sensor specifications, ion imaging measurements with an existing CMOS sensor, VANILLA [13], have been performed. VANILLA does not have sufficient time resolution to resolve individual mass peaks, but these measurements showed that CMOS sensors produced with a similar technology have sufficient sensitivity for this application. The left graph in figure 4 shows a single ion cluster recorded with the VANILLA sensor. The VANILLA pixel size is $25 \times 25 \mu \mathrm{m}^{2}$ so a $3 \times 3$ pixel matrix can be combined into a single $75 \times 75 \mu \mathrm{m}^{2}$ pixel which has a total area similar to the PImMS pixel area. A simple clustering algorithm was employed which required for a seed pixel to exceed a threshold amplitude. Distribution of cluster amplitudes corresponding to different areas is shown in the right graph of figure 4. The horizontal axis is the VANILLA signal in electrons without any correction on the sensor quantum efficiency. Solid lines correspond to single VANILLA pixels, whilst dashed lines correspond to the "composite" $75 \mu \mathrm{m}^{2}$ pixels. Lines of the same color relate to pixel matrices of the same total area. It is apparent that a single $75 \times 75 \mu \mathrm{m}^{2}$ pixel does not contain the whole cluster. Difference between a single $75 \times 75 \mu \mathrm{m}^{2}$ pixel and $3 \times 3$ matrix of $25 \times$ 


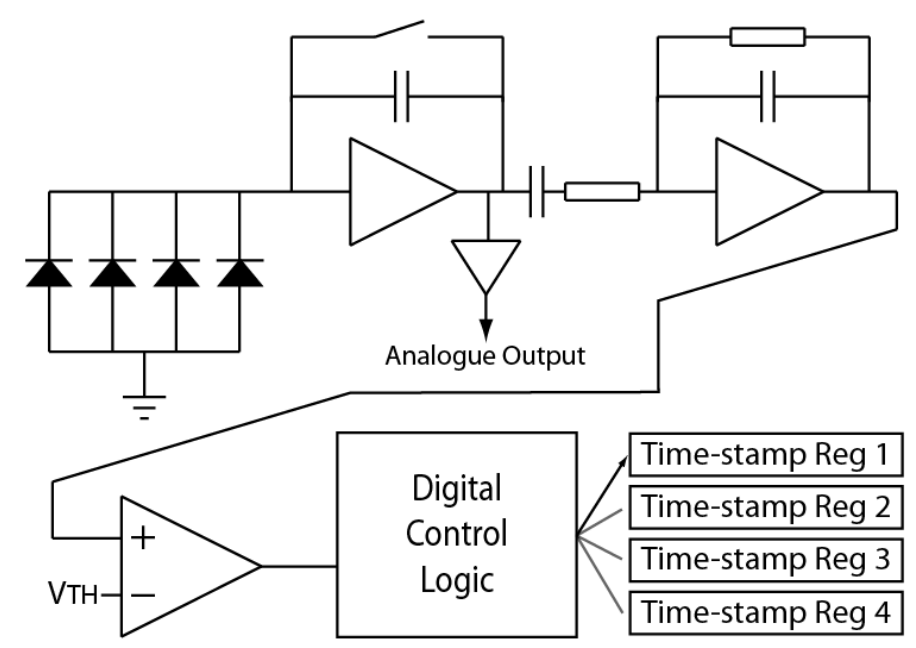

Figure 5. Pixel diagram for the PimMS sensor.

$25 \mu \mathrm{m}^{2}$ pixels is explained by a lower threshold for the seed pixel in the $25 \times 25 \mu \mathrm{m}^{2}$ case. Increasing the area to $7 \times 725 \times 25 \mu \mathrm{m}^{2}$ pixels or to $3 \times 375 \times 75 \mu \mathrm{m}^{2}$ pixels allows full charge collection in the cluster. It should be noted that the cluster size and cluster amplitude are dependent on the MCP bias voltage and also on the optics used to project a $7 \mathrm{~cm}$ diameter phosphor screen on to a smaller sensor. This study used standard settings without any attempt at optimization of these parameters.

A simplified pixel diagram of the PImMS sensor is shown in figure 5. The charge is collected on to multiple nodes and passed to an integrating preamplifier followed by a shaper and comparator. The pixel controller (Digital Control Logic) keeps track of the memory status and determines in which register to store the current time stamp. In addition to the digital readout of the time stamps the sensor also allows analogue readout of the charge collection node.

Timing resolution is one of the most important parameters for the sensor as it determines the mass resolution. In the PImMS sensor it is expected to be of about $100 \mathrm{nsec}$ and will be limited by the time walk for different signal amplitudes. Compared to the TPAC sensor [12] the PImMS front-end electronics will provide an increased dynamic range and response to larger signals.

\section{Summary}

We have described a novel technique, Pixel Imaging Mass Spectrometry, which combines features of TOF-MS and ion imaging, and have presented proof-of-concept results obtained using an offthe-shelf fast framing CCD camera. A specialized CMOS sensor for PImMS is in development with the first prototype expected at the end of 2010 .

\section{Acknowledgments}

The support of the EPSRC through Grant EP/G00224X/1, of the STFC through PNPAS award, of the RC-UK through MI-3 programme (GR/S85733/01) and a 'proof of concept' grant from ISIS Innovation Ltd. are gratefully acknowledged. This work is protected in patents PCT/GB2008/004085 and PCT/GB2004/02014. 


\section{References}

[1] D.W. Chandler and P.L. Houston, Two-dimensional imaging of state-selected photodissociation products detected by multiphoton ionization, J. Chem. Phys. 87 (1987) 1445;

A. Eppink and D.H. Parker, Velocity map imaging of ions and electrons using electrostatic lenses: Application in photoelectron and photofragment ion imaging of molecular oxygen, Rev. Sci. Instrum. 68 (1997) 3477;

B.J. Whitaker (ed.), Imaging in Molecular Dynamics, Cambridge University Press, Cambridge U.K. (2003).

[2] A. Nomerotski et al., Pixel Imaging Mass Spectrometry with fast silicon detectors, in press in Nucl. Instrum. Meth.

[3] ZE-40-04K07, DALSA Corp., http://www.dalsa.com.

[4] L. Lazovsky, D. Cismas, G. Allan and D. Given, CCD sensor and camera for 100 Mfps burst frame rate image capture, Proc. SPIE 5787 (2005) 184.

[5] T.G. Etoh et al., An image sensor which captures 100 consecutive frames at 1000000 frames/s, IEEE Trans. Electron. Dev. 50 (2003) 144.

[6] D.V.T. Son et al., Toward 100 Mega-Frames per Second: Design of an Ultimate Ultra-High-Speed Image Sensor, Sensors 10 (2010) 16.

[7] M. Brouard, E.K. Campbell, A.J. Johnsen, C. Vallance, W.H. Yuen and A. Nomerotski, Velocity map imaging in time of flight mass spectrometry, Rev. Sci. Instrum. 79 (2008) 123115.

[8] R.M. Capriole, T.B. Farmer and J. Gile, Molecular Imaging of Biological Samples: Localization of Peptides and Proteins Using MALDI-TOF MS, Anal. Chem. 69 (1997) 4751;

M. Stoeckli, T.B. Farmer, and R.M. Caprioli, Automated mass spectrometry imaging with a matrix-assisted laser desorption ionization time-of-flight instrument, J. Am. Soc. Mass Spectrom. 10 (1999) 67.

[9] L.J. Frasinski, K. Codling and P.A. Hatherly, Covariance Mapping: A Correlation Method Applied to Multiphoton Multiple Ionization, Science 246 (1989) 1029.

[10] E.R. Fossumb, CMOS image sensors: electronic camera-on-a-chip, IEEE Trans. Electron. Dev. 44 (1997) 1689.

[11] J.A. Ballin et al., Monolithic Active Pixel Sensors (MAPS) in a Quadruple Well Technology for Nearly 100\% Fill Factor and Full CMOS Pixels, Sensors 8 (2008) 5336.

[12] J.P. Crooks et al., A novel CMOS monolithic active pixel sensor with analog signal processing and 100\% fill factor, IEEE Nucl. Sci. Symp. 931 (2007) 931.

[13] S.E. Bohndiek et al., Characterization studies of two novel active pixel sensors, Opt. Eng. 46 (2007) 124003. 